\title{
Physics of Rubber, 1920-1921
}

By W. B. Wiegand

Managing Drrector, Ames Holdfin McCready, Lto, Montreal, Canada

$\mathrm{P}$ ERHAPS the most valuable publication of the period is "Plantation Rubber and the Testing of Rubber," by G. S. Whitby; Part II of which includes a review of the work on rubber by some of the master physicists of the nineteenth century. Nothing could be more fruitful than the repetition of much of this work, with with due regard to composition and life history of the mixings employed.

\section{Physical Structure}

UNSTRAINED-The spatial distribution of discrete ingredients in vulcanized rubber has been admirably studied by $\mathrm{Mr} . \mathrm{H}$. Green, ${ }^{1, *}$ whose photomicrographs show clearly the phenomena of dispersion and agglomeration. The extension of his work to uncompounded rubber may add to our knowledge of its ultimate colloidal structure and so supply visual confirmation or otherwise of the net work hypothesis of C. O. North;2 and of the spheroidal theory of R. W. Lunn. ${ }^{3}$

Strained-The development of vacuoles at the poles of particles in strained rubber, predicted on theoretical grounds by H. F. Schippel, ${ }^{4}$ has been visually confirmed by $\mathrm{H}$. Green. ${ }^{5}$ This phenomenon has deep significance in connection with the theory of reinforcement. ${ }^{6}$

\section{Mechanical Properties}

The physical testing of rubber has been actively carried on but with no important contributions to testing technic. ${ }^{7}$ Standardization as to equipment, procedure, and representation is beginning to receive the attention it deserves. ${ }^{8}$

Stress-STRAIN CuRve - The attention of rubber workers continues to be directed less toward breaking values and more toward the complete curve. The "conchoid" analogy of Schidrowitz and Goldsborough has been shown to be inadequate; ${ }^{9}$ and Shields, ${ }^{10}$ and somewhat later, Hatschek, ${ }^{11}$ have published new formulas based on curves distorted by calculation to actual cross section; the former, who seems to use only the early part of the curve, arriving at a complex logarithmic expression, the latter deriving in elegant fashion a rectangular hyperbola for the same curve. The present writer finds that when the Hatschek method is applied to curves for stocks representing a range of typical factory mixings the construction is inapplicable. Moreover, neither author has established any physical significance for the parameters that appear in their equations.

Vog $t^{12}$ has described a modified method of recording stressstrain data, which facilitates the closer study of reversion phenomena.

ENERGY RELATIONSHIPS-Of the many physical constants used to described the properties of a sample, perhaps the most comprehensive single index is the energy of resilience as measured by the area subtended by the s.-s. curve on the elongation axis. Proposed in June $1920,{ }^{13}$ this quantity has been widely adopted. Gurney and Tavener ${ }^{14}$ describe the energy absorption of various mixings subjected to re-

* Numbers in text refer to References at end of paper. peated stresses, emphasizing the superiority of high-rubber compounds for work (such as in airplanes) requiring a slow rate of energy absorption. An important application to automotive equipment is the use of rubber spring shackles..$^{15}$ Hysteresis losses; as influenced by cure, pigment loading, and cyclic elongation, are described by Wiegand. ${ }^{16 .}$

An interesting fact is the very large increase in resilience induced by the addition of a highly dispersed nonelastic phase such as carbon black.

Volume Changes-The outstanding. contribution is $H$. F. Schippel's $s^{4}$ paper. showing the enormous increases in volume (as much as 100 pèr cent) when highly. pigmented stocks are strained. This has a vital bearing on elastic theory. Sanderson ${ }^{17}$ has studied the thermal expansion of various mixings, finding no discontinuity at the vulcanizing point.

Grain-Van Rossem ${ }^{18}$ has described calender grain, including its disappearance. through heating, which he assigns to the Joule effect. . In this opinion he is opposed by Wiegand ${ }^{19}$ and Lunn $^{3}$ who regard the phenomenon as essentially due to viscositytemperature relationships. (S e e also Skellon. ${ }^{20}$ )

Hardness tests have been thoroughly studied by Gurney, ${ }^{21}$ particularly as applied to the manufacture of rubber-covered rolls.

EFFECT of CoMpounding INGREDIENTS-The connection between fineness of subdivision and reinforcing action has been definitely established.2,6 Further work is necessary to clear up the role played by adhesional forces as distinguished from specific surface. The mechanical properties of vulcanized rubber have been shown to be controllable over very wide ranges through the intelligent use of various fillers. The remarkable reinforcing properties of carbonate of magnesium have been well established by Greider. ${ }^{22}$ Mineral rubber has been the subject of a masterly study by C. O. North. ${ }^{23}$

The important bearing of particle shape upon the phenomena of grain, set, and hysteresis demands further study. ${ }^{24}$

Measurement of Particle Size-The importance of subdivision as a guide to pigment reinforcement has stimulated H. Green ${ }^{25}$ to develop to great precision the direct microscopic method. Using violet light, this worker has been able to measure particles down to 0.2 micron in diameter. $\mathrm{H}$. A. Gardner26 has described a very novel method of estimating the fineness and texture of pigments by rubbing them on a phonograph record. W. W. Vogt ${ }^{27}$ favors the obscurometer method of measuring average particle size. This has had strong criticism from the standpoint of refractive index..$^{28}$

Those who favor clay as a compounding ingredient will find the paper by Comber ${ }^{29}$ very suggestive.

The race for superfine rubber pigments has led to intensive research, working both from the atomic state (sublimed litharge, zinc oxide, magnesium carbonate), and from the coarse condition. ${ }^{30}$

Thus we may soon come upon the ideal pigment, one 